See discussions, stats, and author profiles for this publication at: https://www.researchgate.net/publication/329900108

\title{
Real life experiences in HCV management in 2018
}

Article in Expert Review of Anti-infective Therapy · December 2018

Dol: 10.1080/14787210.2019.1563755

CITATIONS

0

33 authors, including:

Antonio Craxì

Università degli Studi di Palermo

821 PUBlicATIONS 30,177 CITATIONS

SEE PROFILE

6 Maurizia Rossana Brunetto

Università di Pisa

478 PUBLICATIONS 11,743 CITATIONS

SEE PROFILE
READS

197

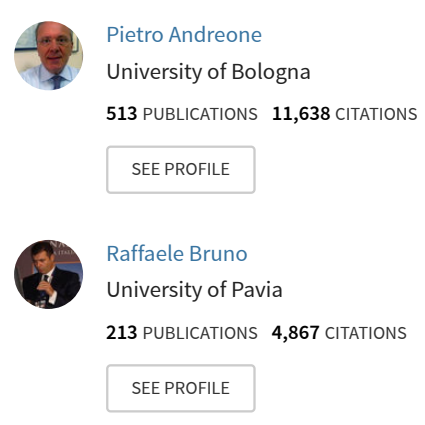

Some of the authors of this publication are also working on these related projects:

Probiotics and health View project

Project HCV RNA infection in mediterranean people View project 


\section{Real life experiences in HCV management in 2018}

Mauro Viganò, Massimo Andreoni, Carlo Federico Perno, Antonio Craxì, Alessio Aghemo, Alfredo Alberti, Pietro Andreone, Sergio Babudieri, Stefano Bonora, Maurizia Rossana Brunetto, Raffaele Bruno, Savino Bruno, Vincenza Calvaruso, Nicola Caporaso, Fabio Cartabellotta, Francesca CeccheriniSilberstein, Valeria Cento, Alessia Ciancio, Piero Colombatto, Nicola Coppola, Vito Di Marco, Giovanni Di Perri, Stefano Fagiuoli, Giovanni Battista Gaeta, Antonio Gasbarrini, Pietro Lampertico, Adriano Pellicelli, Tullio Prestileo, M Puoti, G Raimondo, Giuliano Rizzardini, Gloria Taliani \& Anna Linda Zignego

To cite this article: Mauro Viganò, Massimo Andreoni, Carlo Federico Perno, Antonio Craxì, Alessio Aghemo, Alfredo Alberti, Pietro Andreone, Sergio Babudieri, Stefano Bonora, Maurizia Rossana Brunetto, Raffaele Bruno, Savino Bruno, Vincenza Calvaruso, Nicola Caporaso, Fabio Cartabellotta, Francesca Ceccherini-Silberstein, Valeria Cento, Alessia Ciancio, Piero Colombatto, Nicola Coppola, Vito Di Marco, Giovanni Di Perri, Stefano Fagiuoli, Giovanni Battista Gaeta, Antonio Gasbarrini, Pietro Lampertico, Adriano Pellicelli, Tullio Prestileo, M Puoti, G Raimondo, Giuliano Rizzardini, Gloria Taliani \& Anna Linda Zignego (2018): Real life experiences in HCV management in 2018, Expert Review of Anti-infective Therapy, DOI: 10.1080/14787210.2019.1563755

To link to this article: https://doi.org/10.1080/14787210.2019.1563755

Accepted author version posted online: 24 Dec 2018.

LIII Article views: 1
Submit your article to this journal $\sqsubset$ View Crossmark data $\nearrow$ 
Publisher: Taylor \& Francis

Journal: Expert Review of Anti-infective Therapy

DOI: $10.1080 / 14787210.2019 .1563755$

Article type: Review

\section{Real life experiences in HCV management in 2018}

Mauro Viganò ${ }^{1}$, Massimo Andreoni ${ }^{2}$, Carlo Federico Perno ${ }^{3}$, Antonio Craxi $^{4}$, Alessio Aghemo ${ }^{5}$ Alfredo Alberti ${ }^{6}$, Pietro Andreone ${ }^{7}$, Sergio Babudieri ${ }^{8}$, Stefano Bonora ${ }^{9}$, Maurizia Rossana Brunetto $^{10}$, Raffaele Bruno ${ }^{11}$, Savino Bruno ${ }^{12}$, Vincenza Calvaruso ${ }^{4}$, Nicola Caporaso ${ }^{13}$, Fabio Cartabellotta $^{14}$, Francesca Ceccherini-Silberstein ${ }^{15}$, Valeria Cento ${ }^{3}$, Alessia Ciancio ${ }^{16}$, Piero Colombatto $^{10}$, Nicola Coppola ${ }^{17}$, Vito Di Marco ${ }^{4}$, Giovanni Di Perri ${ }^{9}$, Stefano Fagiuoli ${ }^{18}$, Giovanni Battista Gaeta $^{19}$, Antonio Gasbarrini ${ }^{20}$, Pietro Lampertico ${ }^{21}$, Adriano Pellicelli ${ }^{22}$, Tullio Prestileo ${ }^{23}$, M Puoti $^{24}$, G Raimondo ${ }^{25}$, Giuliano Rizzardini ${ }^{26}$, Gloria Taliani ${ }^{27}$, Anna Linda Zignego ${ }^{28}$

${ }^{1}$ Hepatology Unit, Ospedale San Giuseppe, University of Milan, Milan, Italy.

${ }^{2}$ Department Medicine of Systems University Tor Vergata, Rome, Italy.

${ }^{3}$ University of Milan Department of Laboratory Medicine, Niguarda Hospital, Milan, Italy.

${ }^{4}$ Department of Gastroenterology, DiBiMIS, University of Palermo, Palermo, Italy.

${ }^{5}$ UO Medicina Interna ed Epatologia, Humanitas University and Research Hospital, Pieve Emanuele, Milano, Italy.

${ }^{6}$ Department of Molecular Medicine, University of Padua, Padua, Italy.

${ }^{7}$ Centro per lo Studio e Ricerche delle Epatiti, Dipartimento di Scienze Mediche e Chirurgiche, Università di Bologna, Bologna, Italy.

${ }^{8}$ Infectious Diseases Department, AOU Sassari, University of Sassari, Sassari, Italy.

${ }^{9}$ Unit of Infectious Diseases, Department of Medical Sciences, University of Turin, Turin, Italy.

${ }^{10}$ Dipartimento di Medicina Clinica e Sperimentale Università di Pisa, UO Epatologia Azienda Ospedaliero-Universitaria Pisana, Pisa, Italy.

${ }^{11}$ Division of Infectious Diseases, Fondazione IRCCS Policlinico San Matteo, University of Pavia, Pavia, Italy.

${ }^{12}$ Department of Internal Medicine, Humanitas University Medicine, Rozzano, Italy.

${ }^{13}$ Department of Clinical Medicine and Surgery, Gastroenterology Unit, University of Naples

"Federico II", Naples, Italy. 
${ }^{14}$ Department of Internal Medicine Buccheri La Ferla Hospital Fatebenefratelli Palermo, Italy.

${ }^{15}$ Department of Experimental Medicine and Surgery, University of Rome Tor Vergata, Italy.

${ }^{16}$ Dipartimento di Scienze Mediche, Università di Torino, Torino, Italy.

${ }^{17}$ University of Campania; Infectious Diseases Unit, AORN Caserta, Italy.

${ }^{18}$ USC Gastroenterologia Epatologia e Trapiantologia, Dipartimento di Medicina Specialistica e dei Trapianti, ASST Papa Giovanni XXIII, Bergamo, Italy.

${ }^{19}$ Malattie Infettive, Università della Campania "Luigi Vanvitelli", Napoli, Italy.

${ }^{20}$ Fondazione Policlinico Gemelli IRCCS, Universita' Cattolica del Sacro Cuore, Roma, Italy.

${ }^{21}$ Gastroenterology and Hepatology Division, Fondazione IRCCS Ca' Granda Ospedale Maggiore

Policlinico, Università di Milano, Milano, Italy.

${ }^{22}$ UOC Malattie del Fegato Dipartimento Interaziendale Trapianti Azienda Ospedaliera San Camillo Forlanini Roma, Italy.

${ }^{23}$ Infectious Diseases Unit and Centre for Migration and Health ARNAS, Civico-Benfratelli Hospital, Palermo, Italy.

${ }^{24}$ SC Malattie Infettive, Department of Infectious Diseases, ASST Grande Ospedale Metropolitano Niguarda, Milano, Italy.

${ }^{25}$ Division of Clinical and Molecular Hepatology, Department of Internal Medicine, University Hospital of Messina, Messina, Italy.

${ }^{26}$ Infectious Diseases Department ASST Fatebenefratelli Sacco, School of Clinical Medicine, Faculty of Health Science, University of the Witwatersrand, Johannesburg.

${ }^{27}$ Infectious Diseases Unit and School of Tropical Medicine, Sapienza of Rome University, Rome, Italy.

${ }^{28}$ Department of Experimental and Clinical Medicine and Department of Oncology, Interdepartmental Hepatology Center MASVE, Azienda Ospedaliero-Universitaria Careggi (AOUC), Florence, Italy.

\section{Corresponding author:}

Mauro Viganò

Hepatology Unit, Ospedale San Giuseppe,

University of Milan, Milan, Italy.

Via S. Vittore 12, 20123 Milano, Italy

Tel : +390285994373

Fax : +390285994267

email: mvigano72@gmail.com 


\begin{abstract}
Introduction: Hepatitis $\mathrm{C}$ virus (HCV) infection is a major cause of chronic liver disease, with approximately 71 million chronically infected individuals worldwide. Treatment of chronic hepatitis $\mathrm{C}$ has considerably improved in the last few years thanks to the introduction of direct acting antivirals able to achieve sustained virological response in more than $95 \%$ of patients. Successful anti-HCV treatment can halt liver disease progression and solve the HCV-related extrahepatic manifestations, eventually reducing liver-related and overall mortality.

Areas covered: With the aim to respond to unmet needs in patient's identification, universal access to antiviral therapy and treatment optimization in specific setting of $\mathrm{HCV}$-infected patients, a group of Italian experts met in Stresa in May 2018. The summary of the considerations arising from this meeting and the final statements are reported in this paper.
\end{abstract}

Expert commentary: All the advances on HCV cure may have a real clinical impact not only in individual patients but also at the social health level if they are applied to all infected patients, independently from the stage of liver disease. Further improvements are needed in order to attain $\mathrm{HCV}$ elimination, such as the development of an enhanced screening program working in parallel to the present treatment options.

\title{
Keywords
}

Antiviral therapy, direct acting antivirals, DAAs, hepatitis $\mathrm{C}$ virus, $\mathrm{HCV}$, chronic liver disease 


\section{Article highlights}

- Treatment of chronic hepatitis $\mathrm{C}$ has considerably improved in the last few years thanks to the introduction of DAAs able to achieve SVR in more than $95 \%$ of patients.

- Successful anti-HCV treatment can halt liver disease progression and solve the HCV-related extra-hepatic manifestations, eventually reducing liver-related and overall mortality.

- All the advances on HCV cure may have a real clinical impact at the social health level if they are applied to all infected patients, independently from the stage of liver disease.

- Treating patients with early liver disease today will in fact, in the future, reduce the mortality related to the progression of liver injury and extrahepatic complications, curb the transmission of infection and improve the patients' quality of life.

- In order to attain HCV elimination we should develop and/or enhance screening program both in the general population and in the higher risk patients and linkage to care from all infected patients. 


\section{Introduction}

Hepatitis $\mathrm{C}$ virus (HCV) infection is one of the main causes of chronic liver disease worldwide and is responsible for a large proportion of liver-related deaths, mostly due to hepatocellular carcinoma (HCC) and cirrhosis complications $(1,2)$. Approximately at least 71 million people worldwide are currently infected with $\mathrm{HCV}$, although reliable epidemiological data are elusive in most Countries, including Italy (1). Antiviral treatment of chronic hepatitis $\mathrm{C}(\mathrm{CHC})$ has been revolutionized by the introduction of direct acting antivirals (DAAs) able to achieve sustained virological response (SVR) in more than $95 \%$ of patients. SVR has been associated with the reduction of HCV transmission, improvement of hepatic inflammation and fibrosis, liver function and survival (1). DAAs target key steps of HCV replication and can be divided into 4 classes based on their target and mechanism of action: NS3 protease inhibitors (PIs), NS5B polymerase inhibitors [nucleos(t)ide and nonnucleoside inhibitors, NI and NNI, respectively] and NS5A inhibitors. Combination of different classes of DAAs is essential to achieve SVR.

In Italy, the introduction of DAAs in the clinical practice followed two different phases of reimbursement. In the first, the Italian Medical Agency (AIFA) reimbursed Sofosbuvir (SOF), SOF/Ledipasvir (LDV), SOF/Daclatasvir (DCV), SOF/Simeprevir and Ombitasvir/Paritaprevir/Ritonavir \pm Dasabuvir only for patients with severe hepatic fibrosis or cirrhosis (defined according to liver histology, i.e. $\mathrm{F} \geq 3$ according to METAVIR or $\mathrm{S} \geq 4$ at the Ishak score, and by liver stiffness measurements by Fibroscan $\geq 10 \mathrm{kPa}$ ), with treated HCC, with liver transplant (LT) and with HCV-related extra-hepatic manifestations (EHMs). From March 2017, SOF/Velpatasvir (VEL), Grazoprevir (GZR)/Elbasvir (EBR) and from October 2017 Glecaprevir/Pibrentasvir (GLE/PIB), are reimbursable for all the HCV infected patients, whereas the combination of SOF/VEL/Voxilaprevir (VOX) is reimbursable since April 2018 only for those who have failed a previous DAA-based regimen. 
Among the 71.345 patients treated till March 2017, 45\% had cirrhosis, 23\% F3 fibrosis and 26\% F1-F2 fibrosis. At the end of May 2018, 138.731 patients had been treated with DAAs with the aim to treat 350.000 subjects by 2020 . However, the lack of reliable epidemiological data on HCV prevalence, in general population as well as in patient's categories at higher risk of infection (men who have sex with men, prisoners, people who inject drugs), has not yet allowed to plan a strategy for screening and treatment.

Currently, the approved DAAs in Italy fall into 2 subcategories: the SOF-based regimens including the combination of the NS5B polymerase NI (SOF) plus an NS5A inhibitor (VEL) and the combination of SOF, VEL and a PI (VOX); the non-SOF based regimens including the combination of PI (GZR) plus an NS5A inhibitor (EBR) or the combination of PI (GLE) plus an NS5A inhibitor (PIB). All SOF-based regimens and GLE/PIB show pangenotypic activity, while GZR/EBR is restricted to HCV-1 and 4. SOF/VEL/VOX and GLE/PIB are ribavirin (RBV)-free regimens and SOF/VEL needs RBV in decompensated cirrhotic patients and may be used with RBV in HCV-3 cirrhotic patients. RBV is also required in HCV-1a and HCV-4 patients with HCV RNA $>800.000$ $\mathrm{IU} / \mathrm{ml}$ and/or the presence of specific NS5A polymorphisms treated with GZR/EBR $(3,4)$. However, given that RBV is not devoid of side effects, when possible, RBV-free regimens should be the preferred treatment option (1).

In patients with compensated cirrhosis [Child Pugh Turcotte (CPT) class A], all DAAs can be safely administered, whereas in patients with hepatic impairment (CPT B and C), use of DAAs is feasible but caution should be used (1). Current DAAs show two distinct pharmacological features in cirrhotic patients. The pharmacokinetics (PK) modification of NS5B polymerase inhibitor and NS5A inhibitors, even in CPT B and C patients, are minimal and compatible with expected safety and efficacy (5). On the other hand, plasma concentrations of PIs increase proportionally with the degree of liver impairment, leading to liver function deterioration, hepatic decompensation and 
death. For that reason any DAA regimen that includes PIs needs to be avoided in CTP B and C patients and in those with a previous hepatic decompensation (1).

Kidney function also guides the choice of DAA, indeed it is mandatory to identify patients with a stage $4 / 5$ [estimated glomerular filtration rate (eGFR) $<30 \mathrm{ml} / \mathrm{min} / 1.73 \mathrm{~m}^{2}$ ] of Chronic Kidney Diseases (CKD). Although real-life data have shown a relatively safe profile for SOF in subjects with eGFR $<30 \mathrm{ml} / \mathrm{min} / 1.73 \mathrm{~m}^{2}$, this drug remains still not recommended in such patients, as an increase of up to 20 times of GS-331007, the dephosphorylation-derived SOF nucleoside metabolite, has been reported $(1,6,7)$. GZR/ELB or GLE/PIB are the two combinations of DAAs approved for the treatment of $\mathrm{HCV}$ patients with a stage $4 / 5 \mathrm{CKD}(8,9)$.

Current DAAs show a limited potential for drug to drug interaction (DDI), that are not dependent on metabolic interference, i.e. CYP3A4-mediated, but rather on inhibition of drug transporters, both at intestinal (PgP, BRCP) and hepatic level (OATP1B1, OATP1B3) (1). Although DDIs with some of the most used concomitant drugs have been formally evaluated (proton pump inhibitors, statins, calcium channel blockers, methadone, buprenorphine, immunosuppressant) the wide therapeutic ranges of DAAs protect them from DDIs and facilitates the management of the latter, but knowledge of PK and pharmacodynamics (PD) data are crucial. Moreover, the possible effects of DAAs on concomitant drugs should rely again on PK/PD data. Therefore, management of concomitant drugs with poor or no PK data, i.e. cardiovascular (anti-platelets, anticoagulants, antiarrhythmic), neuroactive (antipsychotics, neuroleptics, antidepressants) or cancer drugs, needs more clinical data to overcome the uncertainty of current recommendations.

\subsection{Statements}

- Due to Italian reimbursement rules these DAA regimens are the standard of care options for HCV-infected patients:

- in DAA-naïve: SOF/VEL, GZR/EBR, GLE/PIB 
- in DAA-experienced: SOF/VEL/VOX, $(S O F+G L E / P I B$ and $S O F+G Z R / E B R$ pending reimbursement)

- $R B V$-free regimens should be the first line treatment when available

- Regimens which include PIs are contraindicated in CPT B and C patients and in those with a previous hepatic decompensation

- CPT A patients are heterogeneous in terms of liver function and therefore PI-containing regimens should be used with caution in patients with borderline surrogate parameters (low albumin and platelets levels) of liver dysfunction

- Regimens which include SOF should not be the first choice in patients with eGFR $<30$ $\mathrm{ml} / \mathrm{min} / 1.73 \mathrm{~m}^{2}$. However, in specific conditions when PIs are contraindicated SOF could be used with caution

- Wide therapeutic ranges of DAAs protect from DDIs but knowledge of PK/PD clinical data are crucial for an appropriate evaluation of the risk

- Although potential for DDIs is limited and manageable, in most cases there is a substantial lack of PK and PD data for several classes of drugs, making uncertain current recommendations

\section{Real life experience with DAAs in Italy}

In order to assess the overall effectiveness of DAAs in Italian real-life, we retrospectively collected the data of three Hepatitis C Registries (Lombardia, Sicilia and Pisa) including all CHC infected patients over 18 years with available SVR data 12 weeks after the end of DAA-based treatment. Stage of liver disease was assessed on clinical ground, by liver biopsy or liver stiffness measurements (LSM) by Fibroscan, considering as cirrhosis LSM $\geq 12 \mathrm{kPa}$. Overall, 10,711 patients were included; the majorities were middle age cirrhotic males, infected with genotype 1 (Table 1). Overall, 10,248 (96\%) patients achieved SVR: 96\% vs 95.5\% vs 90.4\% in patients without 
cirrhosis, with compensated and decompensated cirrhosis, respectively. During the median 9 (0-36) months of follow-up after the end of treatment, 184 (1.8\%) patients developed an HCC: $1.6 \%$ vs $7.3 \%$ in patients with and without SVR, respectively; $68(0.9 \%)$ patients decompensated: $0.9 \%$ vs $2.8 \%$ in patients with and without SVR, respectively and $94(0.9 \%)$ patients died: $0.7 \%$ vs $4.7 \%$ in those with and without SVR, respectively. Despite our collection lacks a long follow-up after the end of treatment it represents an interesting cohort due to the huge number of patients treated in a real practice confirming the effectiveness of DAAs regimens $(10,11)$.

\section{The clinical benefits of treatment with DAAs}

SVR corresponds to the cure of HCV infection and is generally associated with normalization of liver enzymes and improvement or disappearance of liver inflammation and fibrosis in patients without cirrhosis. DAAs have shown high efficacy and safety also in patients with advanced liver disease, in which hepatic fibrosis may regress, and the risk of complications may be reduced after SVR $(1,2)$. Moreover, the virologic response in patients with advanced liver disease has been found to be associated with an improvement of liver function evaluated by CTP and MELD scores, with a concomitant mortality reduction $(10,11)$. Although first data have reported an high rate of HCC incidence and recurrence after DAAs $(12,13)$, most recent data from large cohorts have suggested that HCC incidence in cirrhotic patients with an SVR decreases over time, and do not suggest an increased risk of recurrence, which is instead related to the timing of DAAs after the HCC cure (1419). The predictors of HCC in patients with SVR are: older age, low platelets count, low albumin values, advanced liver fibrosis and higher levels of serum alpha fetoprotein (AFP). On the other hand, cirrhotic patients who fail DAA treatment are a high risk group for HCC development. To date, all cirrhotic patients, regardless of the SVR achievement, should be maintained on surveillance for HCC with semiannual ultrasonography (US) (20).

Moreover, an increasing number of evidences associate DAA treatment with the clinical reduction of the impact of extra-hepatic manifestations (EHMs). Concerning cryoglobulinemic vasculitis, 
there is a general evidence that 90 to $100 \%$ of the subjects acquire an SVR after treatment, although the clinical response can be limited to about one third of the treated subjects $(21,22)$. Concerning the metabolic modification induced by DAAs, there is a clear evidence that after SVR the glycemic control improves in patients with diabetes $(23,24)$, HDL-cholesterol plasma levels increase (25) and carotid atherosclerosis improves (26). The long-term beneficial effect of HCV elimination on hepatic and extra-hepatic morbidity and mortality after treatment with DAA begins to be evident, and it will be even more in the near future.

\subsection{Statements}

- All patients with HCV infection and EHMs should be treated with DAAs because there is a clinical improvement with a reduction of disease progression, except those with limited life expectancy $(<12$ months $)$

\section{DAAs in decompensated cirrhosis}

Treatment of decompensated patients remains a challenge. In both clinical trials and real-life reports, SVR rates progressively decrease with worsening of liver function: from rates $>95 \%$ in CTP A, to $80-90 \%$ in CTP B, and $<75 \%$ in CTP C (27-30). However, this decrease in SVR rates reflects both non virological (death due to liver disease progression) and virological failure. Patients with decompensated cirrhosis non-responders to DAAs usually do not have baseline HCVresistance associated substitutions (RASs) or poorer adherence, compared to responders. A recent report has shown that non-response in such patients can be associated with a number of immunological dysfunctions (elevated basal CXCL-10 serum level compared with responders; NK immunophenotype characterized by a higher proportion of NK cell expressing the natural cytotoxicity receptor NKp30 compared to responders). Even if treated decompensated patients achieving SVR experience less adverse outcome, compared to non-treated and non SVR patients, in some cases the "virological cure" may not prevent disease progression or liver-related mortality 
especially in case of more advanced liver disease. This is usually referred as "the point of no return" where anti-HCV treatment becomes futile. For this reason, DAAs are not required by patients with a limited life expectancy due to liver disease that cannot be remediated by antiviral treatment, LT or another directed therapy (31). However, the definition of this condition in patients not candidates for LT is challenging in the DAA era. In a post hoc analysis, DAAs are associated with significant decrease in mortality risk in patients with decompensated HCV cirrhosis, up to $60 \%$ by the end of the first year. About 4 months after therapy, the risk of death in decompensated patients receiving DAAs became statistically significantly lower than in a control population of untreated patients (32). Moreover, survival of HCV patients with hepatic decompensation may be predicted accurately using a short (90 days) term model based on MELD, sodium, albumin and hepatic encephalopathy (32) or by a recent BE3A score composed of body mass index (BMI), encephalopathy, ascites, ALT and albumin, able to predict clinical improvement to CPT A stage. The BE3A score can be calculated by the sum of its five components each has 1 point when the criterion is met: $\mathrm{BMI}<25$, absence of encephalopathy, absence of ascites, ALT more than $1.5 \mathrm{x}$ upper limits of normal $(\mathrm{ULN}=60 \mathrm{IU} / \mathrm{L})$ and albumin $>3.5 \mathrm{~g} / \mathrm{dL}$. There were no significant differences in BE3A scores of 15 in predicting LT or death. However, a BE3A score of 0 was associated with a $25 \%$ probability of death or a need for LT by week 36 , with a sensitivity of $63 \%$ and a specificity of $75 \%$. A BE3A score $>1$ was associated with $86-100 \%$ specificity of not having LT or death by week 36 (33). This score seems to have essentially a positive predictive value on survival in patients with decompensated liver disease, but it does not allow to accurately predicting liver related mortality. Thus the evaluation of the risk of death at three months in patients with decompensated cirrhosis should be based on a dynamic evaluation of clinical data in the single patient, including MELD, albumin, BMI, ALT levels, sodium, hepatic encephalopathy and ascites and reserved to centers with experience in the management of patients with end stage liver disease. The initial consideration when facing to decompensated cirrhotic patients is whether they are eligible or not for LT. In this latter case $\mathrm{HCV}$ eradication is the only potentially curative option, and therefore it should be 
considered in all the patients. Conversely, in a LT-eligible decompensated patient the antiviral treatment has the goal to eradicate $\mathrm{HCV}$ in order to improve liver function, reduce portal hypertension-related complications and prevent post-LT HCV recurrence. In patients with decompensated liver disease the decision to start DAAs treatment should always be balanced between the potential benefits (high SVR rates in CTP B, reduced liver disease progression, increase in eligibility for $\mathrm{HCC}$ treatment, no increase in $\mathrm{HCC}$ activity after SVR, improvement quality of life [QOL] and reduced mortality on the waiting list, reduced post-LT HCV recurrence), and the potential harms (potentially greater risk of drug toxicity, lower SVR rates compared to postLT antiviral treatment, reduced priority for LT (purgatory effect), possibility to receive HCVpositive graft).

Since SVR rates are reduced in CPT B or C patients, the risk of the emergence of RASs must be taken into account in patients eligible for LT aiming to avoid an unwanted more difficult to treat post-LT recipient. The decision to treat a waitlisted patient with $\mathrm{HCC}$ should be individualized. Potential benefits and harms of antiviral therapy should be considered in the patient-provider shared decision in this setting, treatment to prevent recurrence should bear a lower priority, as highly effective therapies are available post-LT (34). The main issue to take into account when managing a waitlisted patients should be: anticipated time to LT, access to living donor LT, rate of availability of anti-HCV positive donors and waiting list mortality rates. At present, CTP B and C patients with concomitant renal impairment (CKD 4/5) represent a small but highly challenging patient populations. Reports on the HCV treatment in these cases are very limited and there are no published controlled clinical trials. All of the available DAAs combinations (GZR/ELB, GLE/PIB) for patients with CKD 4/5 contain PIs and are therefore contraindicated in patients with decompensated cirrhosis. On the other hand, despite the growing reports on safety and tolerability of SOF in patients with HCV and CKD 4/5, the SOF label limits its use in such patients $(35,36)$. 
Moreover, evidence does not support anti HCV treatment for HCV infected persons with limited life expectancy ( $<12$ months) owing to non-liver related comorbidities.

\subsection{Statements}

- The benefits of DAAs are unlikely to be realized in:

- CPT B or C patients not candidates for LT with a MELD>25 and without any of the following: BMI >25, normal albumin, absence of ascites or of hepatic encephalopathy, elevated ALT or normal sodium

- Patients with limited life expectancy (<12 months) owing to non-liver related comorbidities

- Patients with decompensated cirrhosis DAA regimens should include the combination of SOF plus VEL. Efficacy issues force to consider up to 24 weeks off-label treatment and RBV if tolerable

\subsection{LT-eligible patients}

\subsubsection{Decompensated cirrhosis without HCC}

- $\quad C T P B$ and/or MELD $<20$ on the LT-waiting list without refractory portal hypertensionrelated complications or other exceptions requiring urgent $L T$, should be treated with DAAs

- Child $C$ and or MELD $>25$ or patients with an expected LT-waiting list $<3$ months can be transplanted and treated with DAAs afterwards, in order to simplify treatment and improve its efficacy

- Intermediate MELD (21-25) and/or low MELD scores with refractory portal hypertensionrelated complications should be evaluated by an expert multidisciplinary team on a case-bycase basis with the perspective of delisting

\subsubsection{Decompensated cirrhosis with HCC}


- Decompensated cirrhosis with HCC, with an expected LT-waiting list >3-6 months should be treated with DAAs whereas those with an expected LT-waiting list $<3-6$ months should not be treated with DAAs

\subsection{Decompensated cirrhosis with renal failure}

\subsubsection{Non-LT eligible patients}

- These patients should be managed at a tertiary reference center and the decision whether to treat must be based on a case-by-case basis

- A SOF-based PIs-free combination is at present the only off-label option (dose adjustments and close monitoring are recommended)

- DAAs are not recommended in patients with hepatorenal syndrome

\subsubsection{LT-eligible patients}

- In candidates to combined liver/kidney transplant, DAAs should be deferred to post-LT

\section{HBV/HCV co-infection and DAAs}

Hepatitis B virus (HBV) and $\mathrm{HCV}$ are the most common etiologic factors of chronic liver disease and a major cause of anticipated liver-related mortality worldwide (1,37). Overall, the estimated prevalence of HBV among anti-HCV positive subjects ranges from $2 \%$ to $10 \%$ (37). Patients with HBV and HCV co-infection are at a higher risk of developing liver cirrhosis and HCC and may need either or both anti-HBV and anti-HCV treatments, depending on their viral status, by using therapeutic regimens for $\mathrm{HBV}$ or HCV mono-infection. Preliminary experiments suggested that HCV core protein could suppress HBV replication, however more "physiological" in vitro models proved that $\mathrm{HBV}$ and $\mathrm{HCV}$ can replicate in the same cell without evidence for direct interference (38). Furthermore, a longitudinal study in a cohort of $133 \mathrm{HBV} / \mathrm{HCV}$ co-infected patients showed a 
complex spectrum of virological profiles with major fluctuations of HBV DNA or HCV RNA in one third of the cases (39). Thus, HBV replication also in the setting of HBV/HCV co-infection may have, in the HBeAg-negative phase, spontaneous fluctuations with temporary undetectable HBV DNA and subsequent reactivation. To accurately define the phase of the infection in carriers with low viremia, an adequate monitoring should be undertaken (37). Accordingly, some of the HBV reactivations reported in $\mathrm{HCV}$ co-infected patients treated with DAAs could be spontaneous fluctuations of HBV replication. Interestingly a recent meta-analysis reported a pooled proportion of $\mathrm{HBV}$ reactivation of $24 \%$ (95\%CI: 19-30) in DAAs treated patients, similar to the spontaneous virological fluctuation observed in untreated $\mathrm{HBV} / \mathrm{HCV}$ co-infected carriers (40). Nevertheless, the tight association of $\mathrm{HBV}$ reactivation with DAAs treatment, even if rarely $(1.4 \%, 95 \% \mathrm{CI} 0.8-2.4)$ also in HBsAg negative/anti-HBc positive patients, suggests that a modulation of the innate immune response following HCV clearance could favor a boost of HBV replication $(41,42)$. In most of the cases the increase of HBV DNA serum levels is modest without ALT elevation, but cases of severe ALT flares eventually associated with liver decompensation had been reported $(41,42)$. According to these data, the EASL HBV and HCV clinical practice guidelines $(1,37)$ advise that HBsAg-positive patients undergoing DAAs should be considered for concomitant nucleot(s)ide analogs (NUCs) treatment or prophylaxis. However, it must be noted that most patients with HCV/HBV co-infection with advanced liver disease should be already on treatment with NUCs. HBV reactivations without hepatitis flares had been also reported in patients with occult HBV infection treated with DAAs $(41,42)$. Accordingly, only a careful monitoring without an anti-HBV prophylaxis is recommended $(1,37)$.

\subsection{Statements}

- All patients should be tested for HBsAg, anti-HBc IgG and anti-HBs before DAAs

- Simultaneous administration of NUCs is recommended in patients fulfilling the standard criteria for HBV treatment 
- HBsAg-positive patients undergoing DAAs, but without the standard criteria for HBV treatment should be considered, on single patient basis, according to the severity of liver disease, for concomitant NUCs prophylaxis until week 12 post DAA and further monitored closely

- HBsAg-negative/anti-HBc IgG positive \pm anti-HBs positive patients undergoing DAAs should be tested for HBV reactivation (HBsAg and/or HBV DNA) in case of ALT elevation

\section{HIV/HCV co-infection}

$\mathrm{HIV} / \mathrm{HCV}$ co-infected patients with controlled HIV viremia remain at higher risk to have liver fibrosis progression than HIV mono-infected patients, for this reason is mandatory the treatment of HCV infection. The goal of HCV treatment is to achieve SVR, which is considered to be curative $(1,31)$. Second wave of DAAs have closed the gap in treatment responses previously seen between mono- and co-infected patients. With the current regimens proving equally efficacy for both HCV mono-infected and HIV/HCV co-infected patients, the recommended regimens for co-infected patients are the same as for HCV mono-infected patients, with the additional consideration of potential DDIs with anti-HIV therapies. Shortening therapy ( 8 weeks treatment without RBV) may be feasible and cost-saving only in treatment-naïve patients with F0-2 and baseline HCV RNA $<6$ million IU/ml (43). Active drug, alcohol abuse, neuropsychiatric disease and unstable housing among $\mathrm{HIV} / \mathrm{HCV}$ co-infected persons continue to be ongoing barriers to access to care; however, several studies have presented convincing evidence that these issues are not contraindications to treatment and that patients with these disadvantages do not necessarily have reduced responses to therapy (44).

\subsection{Statements}

- Every person with HIV must be screened for HCV and viceversa 
- Patients with HIV/HCV co-infection should be treated with DAAs regardless of liver disease stage and other concomitant comorbidities (drug abuse, alcoholism, psychiatric disorders)

- Due to DDI between HIV and HCV PIs, a careful checking is needed before to start DDAs

- Patients with HIV at high risk of HCV re-infection should be tested every 6 months for HCV-RNA after achieving SVR

\section{Management of DAA-failures}

Despite the excellent efficacy of DAAs, virological failures can occur and are often associated with development of resistance, with differences according to the type of regimen and HCV genotype $(1,45-47)$. The natural presence of RASs, as well as their rapid emergence during incomplete drug pressure, is intrinsic characteristic of HCV that greatly affect treatment outcome and the chances to achieve a virological cure (48-50). Up today, a high number of RASs in NS3, NS5A, and NS5B have been associated in vivo and/or in vitro with reduced susceptibility to DAAs (51). Guidelines and experts on $\mathrm{HCV}$ treatment agree that understanding more about resistance may help the clinicians to learn why some patients failed, and may allow the optimization of both first and second-line treatment $(1,2,31,52,53)$. The presence of drug-resistance at failure could represent an important issue for re-treatment options, especially when RASs are present in NS5A and/or in multiple DAA-targets. In DAA-failing patients, RASs prevalence and complexity are remarkably high, and in some cases of real life higher than previously reported (46-48). In particular, NS5A RASs at failure are very common (>90\%), also with the new inhibitors VEL and PIB $(54,55)$, and are very fit and tend to persist longer compared to NS3 and NS5B RASs (56). Furthermore, the presence of specific NS5A RASs before a first- and, even more, a second-line NS5A-including regimen can significantly affect the achievement of a SVR in HCV-1a and 3 cirrhotic patients (48$50,54-58)$. 
One proposed and frequently used approach for re-treatment after a virologic failure is to switch the DAA-class in combination with SOF, thanks to its high genetic barrier to resistance. This retreatment strategy had particularly sense when first-line regimen included a NS3 inhibitor. More complex is the situation today, when the majority of patients are using a NS5A inhibitor in first line, and in case of failure, the re-treatment could represent a big challenge. In this scenario, HCV resistance test could represent a helpful tool to characterize the presence of clinical relevant RASs for each specific DAA. In the new EASL guidelines, HCV resistance testing prior to re-treatment in patients who failed any DAA-containing regimens is considered useful to guide re-treatment in the context of a multidisciplinary team including experienced clinicians and virologists (1). Indeed, the resistance testing for all three genes (NS3, NS5A and NS5B) at virological failure after a DAAs was proposed in order to better guide re-treatment decisions (2,53). In addition, direct HCV sequencing in this setting also allows re-evaluating the infecting genotype and subtype.

In cases of absence of RASs the choice of the new re-treatment can be facilitated among different options of regimens. Differently, in cases of high-grade multi-resistance (complex patterns to NS5A class and/or several classes), the combination of 3 or 4 drugs with different mechanisms of action, which affect all 3 genes, increasing the duration of the treatment and adding RBV, can improve the chances of cure (1,31,56-58). Indeed, as recommended by new AASLD 2017 and EASL 2018 guidelines, the most reasonable approach is to re-treat patients with the new combination of SOF/VEL/VOX for 12 weeks (1,31). In case of presence of complex resistance and predictors of lower response (advanced liver disease, multiple courses of DAAs treatment, high viremia) it would be reasonable to evaluate the potential use of GLE/PIB in association with SOF for 12 weeks, and in case of very difficult-to-cure patients it has been recommended to evaluate both treatment options with RBV and/or with the extension of treatment to 16-24 weeks (1).

\subsection{Statements}


- In DAA-exposed patients verify all possible causes of virologic failure: incorrect genotype, poor adherence and consider the possibility of re-infection. HCV resistance should be performed at the time of failure in certified laboratories, on all three genes to account for multiple RASs

- Re-treatment options should be agreed between clinicians and virologists on RASs profile, viral load and patient characteristics

- Off-label solutions (extension of duration, $R B V$ use) can be considered for patients with complex resistance profiles

\section{The burden of HCV infection in Italy: how to bring out the hidden?}

Recently, Morisco et al. (59) assessed the prevalence and risk factors for HCV infection in the adult general population of Naples, selected from three general practitioner patient registers. The antiHCV prevalence was $3 \%$, and $22.5 \%$ of anti-HCV positive subjects were previously unaware of their status. Similar data emerged from the study of Andriulli et al. (60) who assessed the prevalence and risk factors among Italian residents in 5 metropolitan areas. In this study $2.3 \%$ of subjects tested anti-HCV positive and nearly $80 \%$ of anti-HCV positive subjects were aware of their status. In individuals attending the surgeries of 14 general practitioners working in Messina, Cacciola et al. (61) documented HCV infection in 69/1189 (5.8\%) individuals with increased liver enzyme levels. Interestingly, 21/69 (30\%) of anti-HCV individuals were anware of their infection. In our Country major barriers to the potential large use of DAAs have been low rates of HCV screening and diagnosis therefore effective screening programs are urgently needed to provide undiagnosed $\mathrm{HCV}$-infected individuals with therapy and to spread information about preventive measures that should be taken to decrease future morbidity and mortality. In regions where the majority of patients belong to a well-defined age group, birth cohort testing has proven efficacious whereas systematic one-time testing has been recommended in countries with high endemicity 
and/or with the goal of complete eradication. For this reason the optimal regional or national screening approaches should be determined and the screening strategies for HCV infection should be defined according to the local epidemiology of HCV infection. Screening strategies for HCV infection may include screening of populations at risk of infection, birth cohort testing, and general population testing in areas of intermediate to high seroprevalence $(\geq 2 \%-5 \%)(1)$. Universal screening will yield low results and would not be cost-effective in low-prevalence countries where instead the risk-besed screening are more effective. In The US the Centers for Disease Control and Prevention (CDC) and the Preventive Services Task Force recommend screening for HCV for two groups of individuals $(62,63)$ : all individuals born in the years $1945-1965$, based on the finding that approximately $75 \%$ of persons with a positive screening test and chronic HCV infection were born during the years 1945-1965 and in individuals with at least one of the following risk factors: have ever injected illegal drugs; received clotting factor concentrates produced before 1987; received blood or organs before July 1992; have been notified that they received blood from a donor who later tested positive for HCV infection; have ever been on chronic hemodialysis; have a persistently abnormal alanine aminotransferase level; were born to an HCV-positive mother; have HIV infection; have had a needle-stick injury or mucosal exposure to HCV-positive blood (healthcare, emergency medical, and public safety workers).

Screening for HCV infection is performed by measuring antibody to HCV (anti-HCV) in a person's serum. A positive test (detection of the antibody) is not a diagnosis of the disease; it only indicates that a person was previously exposed to the virus. The currently available screening test has a sensitivity of at least $97 \%$ and a specificity of $100 \%$. In addition to enzyme immunoassay (EIA) in serum or plasma, rapid diagnostic tests (RDTs) can be used to screen for anti-HCV antibodies. RDTs use various matrices, including serum and plasma, but also fingerstick capillary whole blood or oral (crevicular) fluid, facilitating screening without the need for venipuncture, tube centrifugation, freezing and skilled labour. RDTs are simple to perform at room temperature 
without specific instrumentation or extensive training (1,64-66). Also dried blood spots can be used to collect whole blood specimens for EIA detection of anti-HCV antibodies in a central laboratory (67-69). If anti-HCV antibodies are detected, the presence of HCV RNA should be determined to identify patients with ongoing infection. Currently, most laboratories use a two-step approach that includes phlebotomy and an antibody test and phlebotomy and subsequently a test for HCV RNA. As a result, a substantial fraction of patients with anti-HCV antibodies never receive confirmatory HCV RNA testing (1). Therefore, reflex testing for HCV RNA should be applied whe never possible when anti-HCV antibodies are detected. The availability of point-of-care tests for HCV infection has the potential to simplify testing algorithms, increase diagnoses, and facilitate linkage to treatment. Moreover, several commercial assays detect HCV RNA (active HCV infection) in 6090 minute. All the further technological advances will enable access to low-cost, rapid, and accurate assays for HCV RNA detection, improving the number of people diagnosed with HCV infection and contributing to global elimination efforts (70-74). Most importantly such assays have the potential to simplify testing algorithms, increase diagnosis rates, and facilitate linkage to treatment, in low- and middle-income areas and in difficult-to reach populations, such as people who inject drugs (PWID). Depending upon relative costs, a direct test for HCV RNA and near-patient testing could be considered to replace screening based on anti-HCV antibody testing by the direct identification of viremic patients. In addition to identifying all the PWID infected patients, the other fundamental task is targeted counseling and risk assessment for men having sex with men (MSM) and inmates to strengthen education programs and to control the spread of the infection. MSM are the third most involved group in HCV transmission, after inmates and PWID, with prevalence up to $4.7 \%$ (75). In recent years, there has been a rapid spread of HCV among MSM, particularly if HIV co-infected (76) and sexual behavior patterns likely drive HCV infection among MSM (77). Unlike other hard to reach populations such as PWID, the fact that HCV infection is more prevalent in HIV-positive MSM represents an advantage, given their link to care, the close monitoring and frequency of test. In this scenario, emerge the importance and the feasibility of HCV treatment 
using DAAs (78). An interesting study on HCV transmission model conducted in UK, suggested that with a scale-up of DAAs use to $80 \%$ regardless of liver disease stage within 1 year from HCV diagnosis, and $20 \%$ every year after, could reduce the prevalence of chronic HCV infection up to $71 \%$ in 2025 , compared to the state of 2015 . An additional value would be given by behavioral interventions, with a further decline of about $2.5 \%$ in the same decade (79).

PWID are "hard-to-reach populations" whereas inmates are difficult to follow over time due to poor adherence and the fact that the latter are often moved from one prison to another not to mention that the services are not well designed to care for person with HCV in prison. Both these patient's categories represent one of most important HCV reservoirs, with a seroprevalence up to $86 \%$ and $84.3 \%$, respectively. These findings suggest the high importance of such special sub-groups for HCV circulation among general population. DAAs could represent an important chance for reducing HCV circulation, and for the treatment of patients who are rarely reached by health-care services.

In a HCV microsimulation model projecting the long-term benefits and costs of different HCV screening and treatment scenarios in US prisons, universal opt-out screening in prisons was highly cost-effective, reducing HCV transmission and associated disease in the outside community (80). Furthermore, a modeling study on HCV transmission among PWID in Scottish prisons, estimated that scaling up HCV treatment to include $80 \%$ of chronically infected PWID that enter the prison system with sentences of sufficient length (more than 12 weeks based on the assumption of 8-week DAA therapy) with an opioid substitution therapy (OST) coverage more than $50 \%$, could reduce the incidence and prevalence of chronic HCV among PWID in Scotland by 55.8\% (95\%CI 49.3$61.4 \%)$ and 55.9\% (95\%CI 51.1-61.3\%), respectively (81). In essence, prison settings and methadone clinics could have a positive impact to screen and treat special subpopulation, normally with poor health-care provision and use. PWID with CHC attending OST community health clinics can achieve adherence and responses to anti-HCV treatment similar to other populations, despite 
injecting drugs at baseline $(82,83)$. PWID should be counselled on the importance of adherence in attaining an SVR. A history of IDU and recent drug use at treatment initiation are not associated with reduced SVR and decisions to treat should be made on a case-by-case basis. PWID with ongoing social issues, history of psychiatric disease and those with more frequent drug use during therapy are at risk of lower adherence and SVR and need to be monitored closely during therapy (84). In PWID we need a combination of strategie sto find those who are infected, get them treated and prevent transmission. However to get out of the HCV epidemic, we need to stop the transmission preventing the primary infection and then prevention of secondary infection, and harm reduction is a key element. Needle syringe program and OST are the primary intervention to reduce HCV transmission among people who inject drugs (85)

In such patients the use of OST, NSP and screening campaigns, associated with the use of DAAs, is cost-effective and represents an exclusive opportunity to reach underserved populations and to reduce $\mathrm{HCV}$ infection to minimal levels (86).

With regards the general population, a web-based model of best medical practice hab beeen showed to improve the management and the treatment of HCV patients with chronic hepatitis and cirrhosis.

\subsection{Statement}

- The universalization of antiviral therapy for HCV infection must be pursued. The linkage to care must be optimized and standardized in order to ensure uniformity throughout the Country

- Italian general practitioners should be sensitized on the benefits for patient and community to treat the largestnumber of infected patientsand therefore they should be involved in disease-specific testing and case finding 
- Sensitization, information and prevention are essential to allow treatment of all patients with HCV infection and to reduce or abolish the transmission of HCV infection in risk groups

- Needle syringe program and OST are the primary intervention to reduce HCV transmission among people who inject drugs

- Inmates, PWID and MSM should be test and treat without delay

- In undocumented migrants coming from low endemic countries universal screening is not recommendedbut direct access to healthcare services and DAAs treatment with transcultural formed staff and transcultural mediators is recommended

- The web-based regional network models for the management of HCV infection must be incentivized as they have proved to be effective in guaranteeing fairness and transparency of treatment but they can also be used to disseminate information to general practitioners to bring out the submerged

\section{Conclusion}

Treatment of CHC has considerably improved in the last few years thanks to the introduction of DAAs able to achieve SVR in more than $95 \%$ of patients. Successful anti-HCV treatment can reduce HCV transmission, halt liver disease progression and solve the HCV-related extra-hepatic manifestations, eventually reducing both liver-related and overall mortality. In Italy, since march 2017 SOF/VEL and GZR/EBR and from October 2017 GLE/PIB are reimbursable for all the HCV infected patients, whereas the combination of SOF/VEL/VOX is reimbursable since April 2018 only for those who have failed a previous DAA-based regimen. The use of DAAs in clinical practice has confirmed the effectiveness and safety of these regimens reported in registration trials thus favoring an extended use in all infected patients.

\section{Expert Opinion}


A number of different studies have shown a clear reduction in the quality of life of CHC-related liver-disease patients. Thse subjects are aware that $\mathrm{CHC}$ can progress to cirrhosis or liver cancer and may experience social isolation, anxiety, and psychological distress, which negatively affected their social lives and family relationship. Longitudinal studies have proved that, following diagnosis, the stigma of liver disease becomes more apparent over time. CHC patients consider their disease shameful and filthy due to the social stigma that hepatitis $\mathrm{C}$ infection is acquired through drug abuse, including narcotics, and risky sexual behaviors. Studies also suggested that these patients are concerned that they may infect the people around them owing to the lack of knowledge on the disease process of hepatitis C. Moreover, they can have bad relationship with their family or be isolated from society because of physical tiredness or feelings of powerlessness. As such, CHC is found to widely affect the patients' personal and family lives and even the society in general, indicating that efforts need to be made to improve their health-related quality of life.

However, in the last years the approach to HCV infection has been dramatically changed following the introduction of the new DAAs. Early improvement in the quality of life of patients who become HCV RNA negative suggests that the virus itself plays a biological role $(88,89)$.

Achievement of SVR with antiviral treatment has been associated with the reduction of HCV transmission, improvement of hepatic inflammation and fibrosis, liver function and survival and their associated costs $(90)$.

DAAs have shown high efficacy and safety also in those patients previously defined as "difficult patients", including those with more advanced liver disease. In such patients the risk of complications and mortality have been reduced after SVR although in some cases the "virological cure" may not prevent disease progression or liver-related mortality as well as in cirrhotic patients obtaining SVR reduces but does not completely eliminate the risk of HCC. However, after an initial phase characterized by the prioritization of the patients with more severe liver disease, necessary to face the costs of the drugs in relation to the high number of treatments required, we are now in a 
phase of universalization of the access to therapy to all infected patients for which the benefit of treatment will be significantly greater. In the near future the number of patients with advances liver disease should gradually disappear and only patients with mild liver disease will remain to be treated.

It is certain that the results achieved so far in CHC patients with the currently approved drugs are excellent and no further regimens are in clinical development. The extension of HCV cure to all infected subjects will further amplify the health benefits while maintaining a favorable cost-benefit ratio. Treating patients with early liver disease will reduce the mortality related to the progression of liver injury and extrahepatic complications, curb the transmission of infection from some persistent reservoirs and improve the patients' quality of life. The reduction in the costs of drugs that have been witnessed in the last year will contribute to the benefits of this therapeutic strategy. However, the advantages of this process of universalization can only be maximized if supported by an effective policy of awareness, information and prevention and if the ability to access treatment will be the same for all patients. The main objective is to establish the tools for a precise epidemiological evaluation, correct information of the categories at risk of contracting and transmitting the infection, enhance screening program both in the general population and in the higher risk patients and an adequate training of healthcare workers.

In the absence of an effective vaccine, treatment of all infected patients is the only strategy that can lead to the elimination of the virus as desired by the World Health Organization (WHO) by 2030. The WHO strategy has a vision of eliminating viral hepatitis as a public health problem and this is encapsulated in the global targets of reducing new viral hepatitis infections by $90 \%$ and reducing deaths due to viral hepatitis by $65 \%$ increasing then testing awarness by $90 \%$ and the number of treated by $80 \%(91)$. 


\section{Funding}

This paper was funded by an unrestricted grant from Gilead Sciences.

\section{Declaration of interest}

M Viganò: Consultant/advisor/sponsored lectures for BMS, Fujirebio, Gilead Sciences, Roche. M Andreoni: Board membership for Gilead Sciences, MSD, ViiV Healthcare, Abbvie, Grants/grants pending for MSD, Payment for lectures including service on speakers bureaus for BMS, Gilead Sciences, Janssen-Cilag, Travel/accommodations/meeting expenses from Abbvie, MSD. C F Perno: grants, research fundings and ad hoc consultation of the following Companies: ViiV, Gilead, MSD, Janssen, Abbvie. A Craxì: Research grants, lecturing fess, advisory boards, scientific consultancy for Novartis, Abbvie, Gilead Sciences, BMS, Achillion, Tibotech, Janssen Cilag, Abbott Diagnostics. A Aghemo: Research Grant Gilead Sciences, AbbVie, Speaker Gilead Sciences, Abbvie, MSD, BMS, Janssen, Alfasigma, Board Gilead Sciences, Abbvie, MSD, BMS, Janssen, Alfasigma. A Alberti: Reaserch grant Gilead Sciences, Abbvie, MSD Speaker Fees Gilead Sciences, Abbvie, MSD, BMS. P Andreone: Advisory Board for MSD, Gilead Sciences, Abbvie, BMS, Intercept Research grant from MSD, Gilead Sciences, Abbvie, BMS. S Bonora: speaker's fees and ad boards fees by MSD, Viiv, Gilead Sciences, Jannssen, Abbvie and BMS. M R Brunetto: Speakers' bureau AbbVie, BMS, Gilead Sciences, Janssen, MSD, Advisory Board AbbVie, Gilead Sciences. V Calvaruso: Speakers' bureau Abbvie, Gilead Sciences, Intercept. R Bruno: Advisory board and speaker Abbvie, BMS, Gilead Sciences, MSD. S Bruno: Speaker bureau MSD. N Caporaso: Research grants, lecturing fees, advisory boards, scientific consultancy for Abbvie, BMS, Gilead Science, Janssen, MSD. F Ceccherini-

Silberstein: Advisory/Consulting/Conferences/Grants: AbbVie, BMS, Gilead Sciences, Janssen, MSD, ViiV. V Cento: Consultant MSD, Speaker's Bureau MSD, Gilead Sciences, Abbvie, BMS. A Ciancio: Speaker bureau MSD, Gilead Science, Abbvie. P Colombatto: Speaker's Bureau e Advisory Boards AbbVie, Janssen, Gilead Science. N Coppola: grants ViiV Healthcare, Janssen- 
Cilag, Gilead Sciences; personal fees from Gilead Sciences, Abbvie, BMS, MSD. G Di Perri:

Speaker and advisor for Abbvie, BMS, MSD, Gilead Sciences, Janssen, ViiV, Pfizer, Novartis, Aventis. V Di Marco: Speaking and advisor for AbbVie, BMS, Gilead Science, MSD, Roche. Grant and research support Abbvie, Bayer, BMS, Gilead, MSD, Roche. S Fagiuoli: Speaker's bureau and Advisory Board for Gilead Science, Abbvie, BMS, MSD, Bayer, Novartis, Kedrion, Alfasigma. G

B Gaeta: Fee as speaker or advisor Abbvie, Gilead Science, MSD, BMS, BioTest, Research support Abbvie, Gilead Science. A Gasbarrini: Advisory board Abbvie, MSD, Alfasigma, Actial, Danone, Sanofi. P Lampertico: Advisory Board/Speaker Bureau for: BMS, Roche, Gilead Science, GSK, Abbvie, MSD, Arrowhead, Alnylam, Janssen. A Pellicelli: Advisory Board Gilead Science. M Puoti: Travel grants and/or fees as a speaker in own events or internal courses and/or as a member of temporary advisory board Abbvie, Gilaed Sciences, MSD, Beckman Coulter. G Raimondo: Grant/research support Gilead Sciences, MSD; Consultant/Advisor: Abbvie, BMS, Gilead Sciences, MSD, Roche; Sponsored lectures: Abbvie, Bayer, BMS, Gilead Sciences, MSD, Roche. G Rizzardini: board speech and research fundings ViiV, Gilead Sciences, Abbvie, MSD, Angelini. G Taliani: Speaking and participation to advisory board for AbbVie, Gilead Sciences, MSD. A L Zignego: consulting or lecturing fees from Gilead Sciences, BMS, MSD, AbbVie. The authors have no other relevant affiliations or financial involvement with any organization or entity with a financial interest in or financial conflict with the subject matter or materials discussed in the manuscript.

\section{Reviewer disclosures}

Peer reviewers on this manuscript have no relevant financial or other relationships to disclose. 


\section{References}

Papers of special note have been highlighted as:

* of interest

** of considerable interest

1) **European Association for the Study of the Liver. EASL recommendations on treatment of hepatitis C 2018. J Hepatol 2018 Apr 9. pii: S0168-8278(18)31968-8. The most up-to-date guidelines on HCV treatment.

2) Viganò M, Perno CF, Craxì A, et al. Treatment of hepatitis C virus infection in Italy: $\mathrm{A}$ consensus report from an expert panel. Dig Liver Dis 2017;49:731-741.

3) Highlights of prescribing information - zepatier ${ }^{\mathrm{TM}}$ (elbasvir and grazoprevir) tablets, for oral use [internet] FDA [cited 21/12/18] available from:

https://www.accessdata.fda.gov/drugsatfda_docs/label/2016/208261Orig1s0001bl.pdf

4) Summary of product characteristics - zepatier $50 \mathrm{mg} / 100 \mathrm{mg}$ film-coated tablets [internet] EMA [cited 21/12/18] Available from:

http://www.ema.europa.eu/docs/en_GB/document_library/EPAR_Product_Information/hum an/004126/WC500211235.pdf

5) Smolders EJ, de Kanter CT, van Hoek B, et al. Pharmacokinetics, efficacy, and safety of hepatitis C virus drugs in patients with liver and/or renal impairment. Drug Saf 2016;39:589-611.

6) Manoi K, Nayak SL, Gupta E, et al. Generic sofosbuvir based direct-acting antivirals in hepatitis C virus infected patients with chronic kidney disease. Liver Int 2018 Apr 20. doi: 10.1111/liv.13863. [Epubaheadofprint]. 
7) Taneja S, Duseja A, De A, et al. Low-dose sofosbuvir is safe and effective in treating chronic hepatitis $\mathrm{C}$ in patients with severe renal impairment or end-stage renal disease. Dig Dis Sci 2018;63:1334-1340.

8) Roth D, Nelson DR, Bruchfeld A, et al. Grazoprevir plus elbasvir in treatment-naive and treatment-experienced patients with hepatitis $C$ virus genotype 1 infection and stage 4-5 chronic kidney disease (the C-SURFER study): a combination phase 3 study. Lancet 2015;386(10003):1537-45.

9) Kosloski MP, Zhao W, Marbury TC, et al. Effects of renal impairment and hemodialysis on the pharmacokinetics and safety of the Glecaprevir and Pibrentasvir combination in hepatitis C virus-negative subjects. Antimicrob Agents Chemother 2018;62(3).

10) Cheung MCM, Walker AJ, Hudson BE, et al. HCV Research UK. Outcomes after successful direct-acting antiviral therapy for patients with chronic hepatitis $\mathrm{C}$ and decompensated cirrhosis. J Hepatol 2016;65:741-747.

11) Backus LI, Belperio PS, Shahoumian TA, et al. Direct-acting antiviral sustained virologic response: impact on mortality in patients without advanced liver disease. Hepatology 2018. [Epub ahead of print].

12) Reig M, Mariño Z, Perelló C, et al. Unexpected high rate of early tumor recurrence in patients with HCV-related HCC undergoing interferon-free therapy. J Hepatol 2016;65: $719-726$.

13) Conti F, Buonfiglioli F, Scuteri A, et al. Early occurrence and recurrence of hepatocellular carcinoma in HCV-related cirrhosis treated with direct-acting antivirals. J Hepatol $2016 ; 65: 727-733$.

14)**Kanwal F, Kramer J, Asch SM, et al. Risk of hepatocellular cancer in HCV patients treated with direct-acting antiviral agents. Gastroenterology 2017;153:996-1005. In patients treated with DAA, SVR was associated with a considerable reduction in the risk of HCC. 
15) Ioannou GN, Green PK, Berry K. HCV eradication induced by direct-acting antiviral agents reduces the risk of hepatocellular carcinoma. J Hepatol 2018;68:25-32.

16) ANRS collaborative study group on hepatocellular carcinoma (ANRS CO22 HEPATHER, CO12 CirVir and CO23 CUPILT cohorts). Lack of evidence of an effect of direct acting antivirals on the recurrence of hepatocellular carcinoma: Data from three ANRS cohorts. $\mathrm{J}$ Hepatol 2016;65:734-740

17) Romano A, Angeli P, Piovesan S, et al. Newly diagnosed hepatocellular carcinoma in patients with advanced hepatitis $\mathrm{C}$ treated with DAAs: a prospective population study. J Hepatol 2018 [Epubahead of print].

18)**Calvaruso V, Cabibbo G, Cacciola I, et al. Incidence of hepatocellular carcinoma in patients with $\mathrm{HCV}$-associated cirrhosis treated with direct-acting antiviral agents.

Gastroenterology 2018 Apr 12. pii: S0016-5085(18)30441-4. doi:

10.1053/j.gastro.2018.04.008. [Epub ahead of print] In this large prospective cohort of patients with HCV-associated compensated or decompensated cirrhosis, SVR to DAAs reduced the incidence of $\mathrm{HCC}$.

19) Cabibbo G, Petta S, Calvaruso V, et al. Is early recurrence of hepatocellular carcinoma in $\mathrm{HCV}$ cirrhotic patients affected by treatment with direct-acting antivirals? A prospective multicentre study.Aliment Pharmacol Ther. 2017;46:688-695.

20) EASL Clinical Practice Guidelines: Management of hepatocellular carcinoma. European Association for the Study of the Liver. J Hepatol 2018, in press

21) Cacoub P, Desbois AC, Comarmond C, et al. Impact of sustained virological response on the extrahepatic manifestations of chronic hepatitis C: a meta-analysis. Gut 2018 Apr 27. pii: gutjnl-2018-316234.

22) Emery JS, Kuczynski M, La D, et al. Efficacy and safety of direct acting antivirals for the treatment of mixed cryoglobulinemia. Am J Gastroenterol 2017;112:1298-1308. 
23) Hum J, Jou JH, Green PK, et al. Improvement in glycemic control of type 2 diabetes after successful treatment of hepatitis C virus. Diabetes Care 2017;40:1173-1180.

24) Ciancio A, Bosio R, Bo S, et al. Significant improvement of glycemic control in diabetic patients with $\mathrm{HCV}$ infection responding to direct-acting antiviral agents. J Med Virol 2018;90:320-327.

25) Sun HY, Cheng PN, Tseng CY, et al. Favouring modulation of circulating lipoproteins and lipid loading capacity by direct antiviral agents grazoprevir/elbasvir or ledipasvir/sofosbuvir treatment against chronic HCV infection. Gut 2017 Jun 14. pii: gutjnl-2017-313832.

26) Petta S, Adinolfi LE, Fracanzani AL, et al. Hepatitis C virus eradication by direct-acting antiviral agents improves carotid atherosclerosis in patients with severe liver fibrosis. J Hepatol 2018 Mar 2. In press

27) Foster GR, Irving WL, Cheung MC, et al. Impact of direct acting antiviral therapy in patients with chronic hepatitis C and decompensated cirrhosis. J Hepatol 2016;64:12241231.

28) Welzel TM, Petersen J, Herzer K, et al. Erratum: daclatasvir plus sofosbuvir, with or without ribavirin, achieved high sustained virological response rates in patients with HCV infection and advanced liver disease in a real-world cohort. Gut 2016;65:2060.

29) Cheung MC, Walker AJ, Hudson BE, et al. Outcomes after successful direct-acting antiviral therapy for patients with chronic hepatitis $\mathrm{C}$ and decompensated cirrhosis. J Hepatol $2016 ; 65: 741-747$.

30) Afdhal N, Everson GT, Calleja JL al. Effect of viral suppression on hepatic venous pressure gradient in hepatitis C with cirrhosis and portal hypertension. J Viral Hepat 2017;24:823831. 
31) AASLD/IDSA HCV Guidance Panel. Hepatitis C guidance: AASLD-IDSA HCV Guidance: Recommendations for testing, managing, and treating Hepatitis $\mathrm{C}$ https://www.hcvguidelines.org/references/aasld-idsa-2017

32) Kim WR, Mannalithara A, Lee $\mathrm{H}$ et al. Survival benefit of direct-acting antiviral therapy in patients with decompensated cirrhosis. 68th Annual Meeting of the American Association for the Study of Liver diseases, October 20-24, 2017, Washington DC, USA; abstract LB-27

33) El-Sherif O, Jiang Z, Tapper E, et al. Baseline factors associated with improvements in decompensated cirrhosis after direct-acting antiviral therapy for HCV infection, Gastroenterology 2018, doi: 10.1053/j.gastro.2018.03.022. In press

34) Terrault NA, McCaughan GW, Curry MP et al. International Liver Transplantation Society: Consensus Statement on Hepatitis C Management in Liver Transplant Candidates. Transplantation 2017;101:945-955

35) Desnoyer A, Pospai D, Le MP, et al. Pharmacokinetics, safety and efficacy of a full dose sofosbuvir-based regimen given daily in hemodialysis patients with chronic hepatitis C. J Hepatol 2016;65:40-47.

36) Saxena V, Koraishy FM, Sise ME, et al. Safety and efficacy of sofosbuvir-containing regimens in hepatitis C-infected patients with impaired renal function. Liver Int 2016;36:807-816.

37) European Association for the Study of the Liver. EASL 2017 Clinical Practice Guidelines on the management of hepatitis B virus infection. J Hepatol 2017;67:370-398.

38) Bellecave P, Gouttenoire J, Gajer M et al. Hepatitis B and C virus co-infection: a novel model system reveals the absence of direct viral interference. Hepatology 2009;50:46-55. 
39) Raimondo G, Brunetto MR, Pontisso P et al. Longitudinal evaluation reveals a complex spectrum of virological profiles in hepatitis B virus/hepatitis C virus-coinfected patients. Hepatology 2006;43:100-7.

40) Mücke MM, Backus LI, Mücke VT et al. Hepatitis B virus reactivation during direct-acting antiviral therapy for hepatitis C: a systematic review and meta-analysis. Lancet Gastroenterol Hepatol 2018 Jan 19. pii: S2468-1253(18)30002-5.

41) Liu CJ, Chuang WL, Sheen IS et al. Efficacy of ledipasvir and sofosbuvir treatment of HCV infection in patients coinfected with HBV. Gastroenterology 2018;154:989-997.

42) Calvaruso V, Craxì A. HBV recurrence after HCV clearance on DAAs: Sometimes they come back.J Hepatol. 2017;67:898-901.

43) The EACS Treatment Guidelines 9.0 October 2017 available: http://www.eacsociety.org/guidelines/eacs-guidelines/eacs-guidelines.html

44) Cachay E, Wyles D, Hill L, et al. The impact of direct-acting antivirals in the hepatitis Csustained viral response in human immunodeficiency virus-infected patients with ongoing barriers to care. Open Forum Infect Dis 2015;2(4):ofv168

45) Di Maio VC, Cento V, Aragri M, et al. Frequent NS5A and multiclass resistance in almost all HCV genotypes at DAA failures: what are the chances for second-line regimens? J Hepatol 2018;68:597-600

46) Dietz J, Susser S, Vermehren J, et al. European HCV resistance study group. Patterns of resistance-associated substitutions in patients with chronic $\mathrm{HCV}$ infection following treatment with direct-acting antivirals. Gastroenterology 2018;154:976-988.e4.

47) Di Maio VC, Cento V, Lenci I, et al. Multiclass HCV resistance to direct-acting antiviral failure in real-life patients advocates for tailored second-line therapies. Liver Int 2016;00:115. 
48) Sarrazin C, Dvory-Sobol H, Svarovskaia ES et al. Prevalence of resistance-associated substitutions in HCV NS5A, NS5B, or NS3 and outcomes of treatment with ledipasvir and sofosbuvir. Gastroenterology 2016;151:501-512.

49) Zeuzem S, Mizokami M, Pianko S, et al. NS5A resistance-associated substitutions in patients with genotype 1 hepatitis $\mathrm{C}$ virus: Prevalence and effect on treatment outcome. $\mathrm{J}$ Hepatol 2017;66:910-918.

50) Harrington PR, Komatsu TE, Deming DJ, et al. Impact of hepatitis C virus polymorphisms on direct-acting antiviral treatment efficacy: regulatory analyses and perspectives. Hepatology 2017 Dec 1. doi: 10.1002/hep.29693.

51) Sorbo MC, Cento V, Di Maio VC, et al. Hepatitis C virus drug resistance associated substitutions and their clinical relevance: Drug Resist Updat 2018 Apr 18. pii: S13687646(18)30024-4.

52) Sarrazin C. The importance of resistance to direct antiviral drugs in HCV infection in clinical practice. J Hepatol 2016;64:486-504.

53) Craxi A, Perno CF, Vigano M, et al. From current status to optimization of HCV treatment: recommendations from an expert panel. Dig Liver Dis 2016;48:995-1005.

54) Foster GR, Afdhal N, Roberts SK, et al. Sofosbuvir and velpatasvir for HCV genotype 2 and 3 infection. N Engl J Med 2015;373:2608-2617.

55) Puoti M, Foster GR, Wang S, et al. High SVR12 with 8-week and 12-week glecaprevir/pibrentasvir: integrated analysis of HCV genotype 1-6 patients without cirrhosis. J Hepatol 2018 Mar 15.

56) Struble K, Chan-Tack K, Qi K, et al. Benefit-Risk assessment for sofosbuvir/velpatasvir/voxilaprevir based on patient population and HCV genotype: FDA's Evaluation. Hepatology 2017 Oct 23. 
57) Poordad F, Pol S, Asatryan A, et al. Glecaprevir/Pibrentasvir in patients with hepatitis C virus genotype 1 or 4 and past direct-acting antiviral treatment failure. Hepatology 2018;67:1253-1260.

58) Wyles D, Weiland O, Yao B, et al. Retreatment of patients who failed glecaprevir/pibrentasvir treatment for hepatitis C virus infection. J Hepatol 2018 vol. 68 PS040

59) Morisco F, Loperto I, Stroffolini T, et al. Prevalence and risk factors of HCV infection in a metropolitan area in southern Italy: Tail of a cohort infected in past decades. J Med Virol 2017;89:291-297.

60) Andriulli A, Stroffolini T, Mariano A, et al. Declining prevalence and increasing awareness of HCV infection in Italy: A population-based survey in five metropolitan areas. Eur J Intern Med 2018 Feb 20.

61) Cacciola I, Scoglio R, Alibrandi A, et al. Evaluation of liver enzyme levels and identification of asymptomatic liver disease patients in primary care. Intern Emerg Med 2017;12:181-186.

62) Smith BD, Morgan RL, Beckett GA, et al. Recommendations for the identification of chronic hepatitis C virus infection among persons born during 1945-1965. MMWR Recomm Rep 2012;61:1-32.

63) Moyer VA. Screening for hepatitis C virus infection in adults. U.S. Preventive Services Task Force Recommendation Statement. Ann Intern Med 2013;159:349-357

64) Chevaliez S, Poiteau L, Rosa I, et al. Prospective assessment of rapid diagnostic tests for the detection of antibodies to hepatitis $\mathrm{C}$ virus, a tool for improving access to care. Clin Microbiol Infect 2016;22:459,e451-e456.

65) Khuroo MS, Khuroo NS, Khuroo MS. Diagnostic accuracy of point-ofcare tests for hepatitis C virus infection: a systematic review and metaanalysis. PLoS One 2015;10:e0121450. 
66) Shivkumar S, Peeling R, Jafari Y, Joseph L, Pant Pai N. Accuracy of rapid and point-of-care screening tests for hepatitis C: a systematic review and meta-analysis. Ann Intern Med 2012;157:558-566.

67) Poiteau L, Soulier A, Rosa I, et al. Performance of rapid diagnostic tests for the detection of antibodies to hepatitis $\mathrm{C}$ virus in whole blood collected on dried blood spots. J Viral Hepat 2016;23:399-401.

68) Soulier A, Poiteau L, Rosa I, et al. Dried blood spots: a tool to ensure broad access to hepatitis C screening, diagnosis, and treatment monitoring. J Infect Dis 2016;213:10871095.

69) Tuaillon E, Mondain AM, Meroueh F, et al. Dried blood spot for hepatitis C virus serology and molecular testing. Hepatology 2010;51:752-758.34-36

70) Chapko MK, Dufour DR, Hatia RI, Drobeniuc J, Ward JW, Teo CG. Cost effectiveness of strategies for testing current hepatitis C virus infection. Hepatology 2015;62:1396-1404.

71) Hirsch AA, Lawrence RH, Kern E, Falck-Ytter Y, Shumaker DT, Watts B. Implementation and evaluation of a multicomponent quality improvement intervention to improve efficiency of hepatitis C screening and diagnosis. Jt Comm J Qual Patient Saf 2014;40:351-357.

72) Snow K, Scott N, Clothier HJ, MacLachlan JH, Cowie B. Limited provision of diagnostic services to Victorians living with hepatitis C antibodies, 2001-2012: a multi-level modelling analysis. Aust N Z J Public Health 2016. doi: 10.1111/1753-6405.12560

73) Treloar C1, Newland J, Harris M, Deacon R, Maher L. A diagnosis of hepatitis C: Insights from a study on patients' experiences. Aust Fam Physician. 2010;39:589-92.

74) Grebely J, Applegate TL, Cunningham P, Feld JJ. Hepatitis C point-of-care diagnostics: in search of a single visit diagnosis. Expert Rev Mol Diagn 2017;17:1109-1115.

75) Falla AM, Hofstraat SHI, Duffell E,et al. Hepatitis B/C in the countries of the EU/EEA: a systematic review of the prevalence among at-risk groups. BMC Infect Dis 2018;18:79. 
76) Bradshaw D, Matthew G, Danta M. Sexually transmitted hepatitis C infection: the new epidemic in MSM? Current Opinions in Infectious Diseases 2013;26:66-72.

77) MacGregor L, Martin NK, Mukandavire C, et al. Behavioural, not biological, factors drive the HCV epidemic among HIV-positive MSM: HCV and HIV modelling analysis including HCV treatment-as-prevention impact. Int J Epidemiol 2017;46:1582-1592.

78) Martin NK, Vickerman P, Dore GJ, et al. The hepatitis C virus epidemics in key populations (including people who inject drugs, prisoners and MSM): the use of direct-acting antivirals as treatment for prevention. Curr Opin HIV AIDS 2015;10:374-80.

79) Martin NK, Thornton A, Hickman M, et al. Can hepatitis C virus (HCV) direct-acting antiviral treatment as prevention reverse the HCV epidemic among men who have sex with men in the United Kingdom? Epidemiological and Modeling Insights. Clin Infect Dis 2016;62:1072-1080.

80) He T, Li K, Roberts MS, et al. Prevention of Hepatitis C by screening and treatment in U.S. prisons. Ann Intern Med 2016;164:84-92.

81) Stone J, Martin NK, Hickman M, et al. Modelling the impact of incarceration and prisonbased hepatitis $\mathrm{C}$ virus (HCV) treatment on $\mathrm{HCV}$ transmission among people who inject drugs in Scotland. Addiction 2017;112:1302-1314.

82) Grebely J, Alavi M, Micallef M, et al. Treatment for hepatitis C virus infection among people who inject drugs attending opioid substitution treatment and community health clinics: the ETHOS Study. Addiction. 2016;111:311-9.

83) Grebely J, Dalgard O, Cunningham EB, et al Efficacy of response-guided directly observed pegylated interferon and self-administered ribavirin for people who inject drugs with hepatitis C virus genotype 2/3 infection: The ACTIVATE study. Int J Drug Policy. 2017;47:177-186. 
84) Grebely J, Robaeys G, Bruggmann P, et al. Recommendations for the management of hepatitis C virus infection among people who inject drugs. Int J Drug Policy. 2015;26: $1028-1038$.

85) Platt L, Minozzi S, Reed J, et al. Needle syringe programmes and opioid substitution therapy for preventing hepatitis $C$ transmission in people who inject drugs. Cochrane Database Syst Rev. 2017;9:CD012021

86) Fraser H, Martin NK, Brummer-Korvenkontio H, et al. Model projections on the impact of $\mathrm{HCV}$ treatment in the prevention of HCV transmission among people who inject drugs in Europe. J Hepatol 2018;68:402-411.

87) Cartabellotta F, Di Marco V; RESIST - HCV. The HCV Sicily Network: a web-based model for the management of HCV chronic liver diseases. Eur Rev Med Pharmacol Sci. 2016;20(1 Suppl):11-16.

88) Younossi ZM, Stepanova M, Henry L, et al. Sofosbuvir and ledipasvir are associated with high sustained virologic response and improvement of health-related quality of life in East Asian patients with hepatitis C virus infection. J Viral Hepat. 2018 Jul 4. doi: 10.1111/jvh.12965. [Epub ahead of print]

89) Younossi ZM, Stepanova M, Reddy R, et al. Viral eradication is required for sustained improvement of patient-reported outcomes in patientswith hepatitis C. Liver Int. 2018 Jun 12. doi: 10.1111/liv.13900. [Epub ahead of print]

90) Younossi ZM, Tanaka A, Eguchi Y, et al. Treatment of hepatitis C virus leads to economic gains related to reduction in cases of hepatocellular carcinoma and decompensated cirrhosis in Japan. J Viral Hepat. 2018 Aug;25(8):945-951.

91) Global Hepatitus Report 2017 [internet] 2017 [cited 21/12/18] Available from: https://www.who.int/hepatitis/publications/global-hepatitis-report2017/en/ 


\section{Table Legend}

Table 1: Baseline clinical, demographic and virological characteristics of 10,711 patients with available SVR12 data after DAAs treatment. 


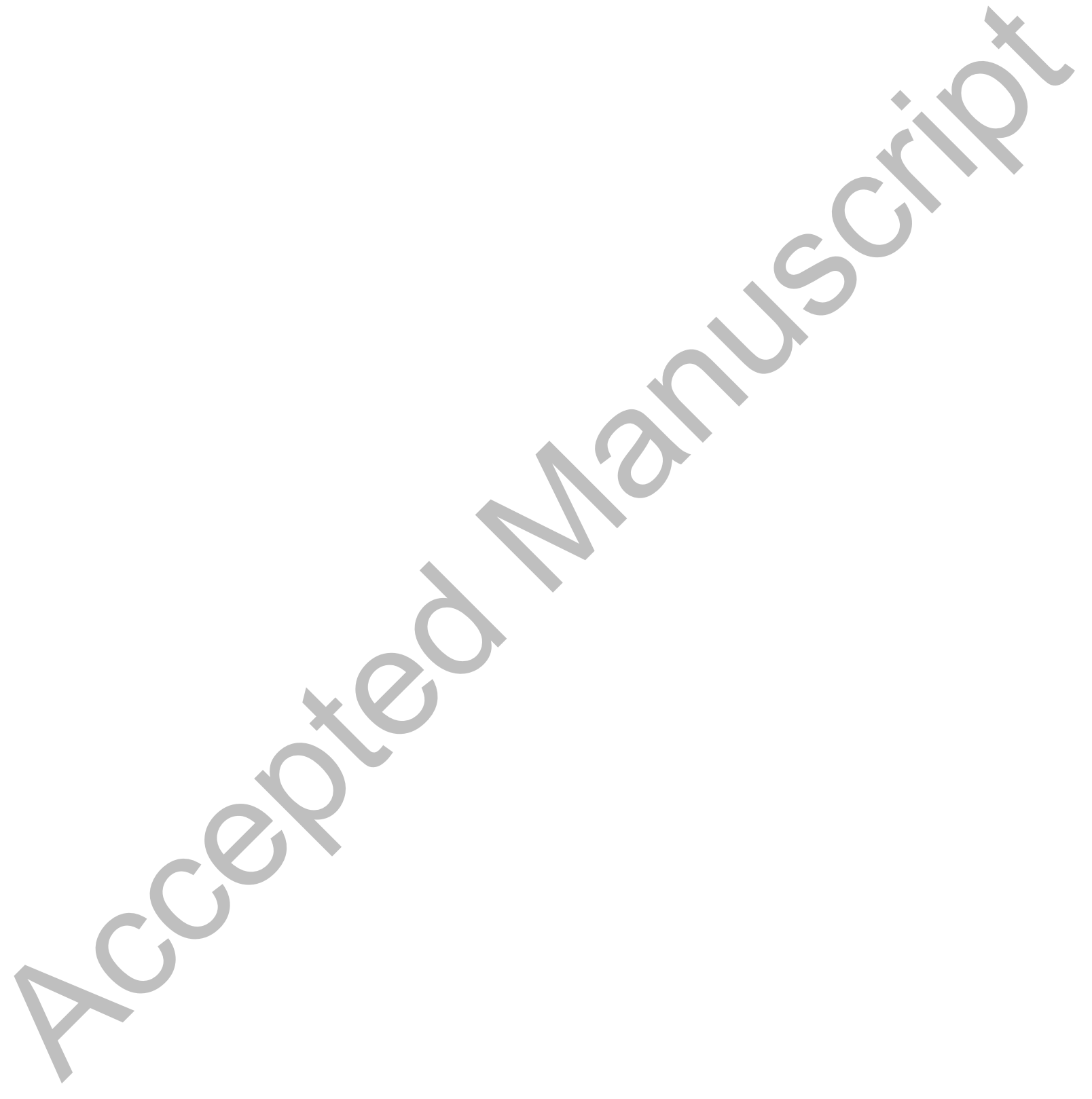


Table- Baseline clinical, demographic and virological characteristics of 10,711 patients with available SVR12 data after DAAs treatment.

\begin{tabular}{|c|c|}
\hline \multicolumn{2}{|l|}{ Characteristics } \\
\hline Age, yrs* & $62(18-88)$ \\
\hline Age $\geq 65$ years & $4644(43 \%)$ \\
\hline Male, n (\%) & $6459(60 \%)$ \\
\hline Genotype, n (\%) & \\
\hline $1 \mathrm{a}$ & $1465(14 \%)$ \\
\hline $1 b$ & $5788(54 \%)$ \\
\hline 2 & $(12 \%)$ \\
\hline 3 & $1217(11 \%)$ \\
\hline 4 & $863(8 \%)$ \\
\hline Others or not available & $99(1 \%)$ \\
\hline Compensated cirrhosis, $\mathrm{n}(\%)$ & $7121(66 \%)$ \\
\hline Decompensated cirrhosis & $714(7 \%)$ \\
\hline $\mathrm{BMI}^{*}, \mathrm{~kg} / \mathrm{m}^{2}$ & $25(14-48)$ \\
\hline ALT, IU/L* & $74(6-1178)$ \\
\hline INR* & $1.06(0.8-3.6)$ \\
\hline Platelets $\times 10^{3} / \mathrm{mm}^{3} *$ & $135(12-1151)$ \\
\hline Albumin, $\mathrm{g} / \mathrm{dL}^{*}$ & $4.0(2.0-5.6)$ \\
\hline Bilirubin, mg/dL* & $0.84(0.2-31)$ \\
\hline Creatinine, $\mathrm{mg} / \mathrm{dL}^{*}$ & $0.8(0.2-9.7)$ \\
\hline
\end{tabular}

